\title{
Simulation and Digital Prototyping of Tangible User Interfaces
}

\author{
Stefan Diewald ${ }^{1}$, Andreas Möller ${ }^{1}$, Luis Roalter ${ }^{1}$, Matthias Kranz ${ }^{2}$ \\ ${ }^{1}$ Distributed Multimodal Information Processing Group, Technische Universität München \\ ${ }^{2}$ Lehrstuhl für Informatik mit Schwerpunkt Eingebettete Systeme, Universität Passau
}

\begin{abstract}
Physical prototyping is an important part in research and development of tangible user interfaces (TUIs). On the way from the idea to a working prototype, hardware prototypes usually have to be crafted repeatedly in numerous iterations. However, this can be very time- and cost-intensive when non-standard, expensive hardware components are part of the design. This brings us to think about digital prototypes that exhibit the same functionality as a physical TUI, but reduce the amount of resources that have to be spent. For that reason, we present a middleware-based process that allows developing and testing fully functional implementations of a tangible user interface as a digital device in a $3 \mathrm{D}$ virtual environment.
\end{abstract}

\section{Introduction}

The development and evaluation of prototypic tangible user interfaces (TUIs) (Ishii \& Ullmer 1997) consumes a lot of effort and time due to iterative design and debug processes on some kind of hardware. Starting from I/O cubes, to tabletops and various augmented everyday objects, each TUI consists of individual hardware that has often to be built from scratch. In order to reduce the development time for initial prototypes, hardware frameworks, such as Blades \& Tiles (Sankaran et al. 2009) or Pin \& Play (Van Laerhoven et al. 2003), are used by developers. However, still a lot of work has to be spent on the hardware before a running prototype can actually be used for evaluating user interaction and HCI-related aspects. This is especially the case for TUIs that are based on novel hardware that is not yet available or cannot be realized within reasonable expenditure.

Hence, a prototyping approach allowing the simulation of tangible user interfaces at an early stage, e.g. to evaluate novel interaction concepts before building any kind of hardware, could extremely shorten the overall development time. 


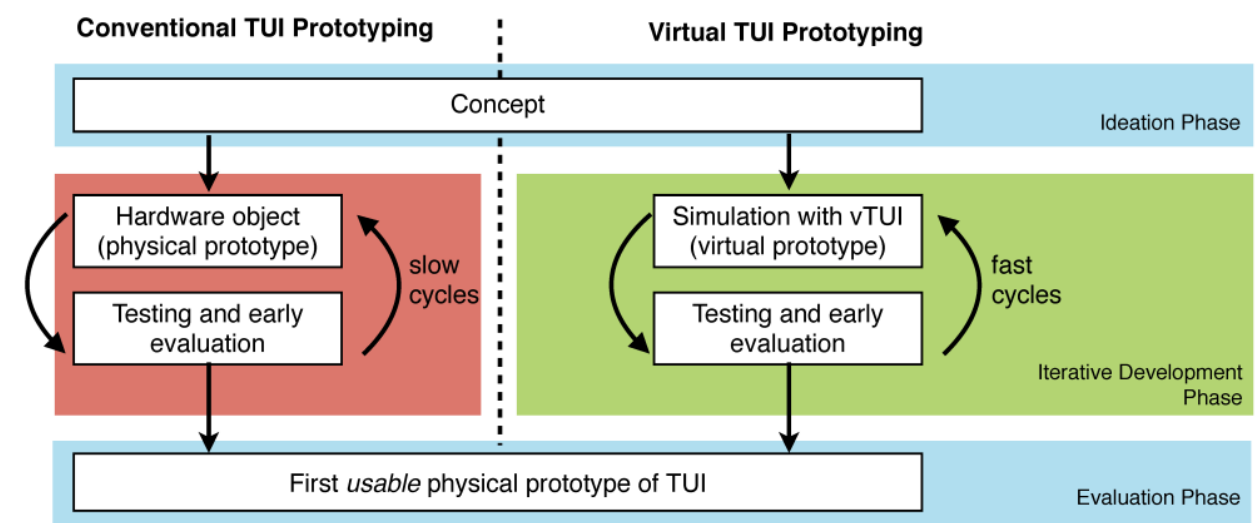

Figure 1: Comparison of the prototyping with physical (left) and digital (right) devices from the idea to the first physical prototype that can be used for a user evaluation. As potentially multiple physical prototypes are needed when prototyping with physical hardware, development cycles are faster with digital prototyping.

In this paper, we introduce a toolkit for TUI simulation that allows shifting the early prototyping process into a high-fidelity 3D virtual environment. That way, shapes of objects for a new TUI and/or new interaction concepts can be evaluated before an actual hardware prototype needs to be built (Holleis et al. 2005). The proposed toolkit is based on a middleware that can be used for digital as well as for physical TUIs. For that reason, interactions between real and simulated components are possible. Moreover, the transition from the digital to the physical prototype does not entail significant changes from software side.

\section{Middleware-based Digital TUI Prototyping}

Based on the results from Roalter et al. with intelligent and smart environments (Roalter et al. 2011), we assembled a toolkit that allows a complete virtual representation of a TUI. As presented in Fig. 2, we can create high-fidelity virtual prototypes that look almost identical to subsequently developed physical prototypes.

\subsection{Requirements for a Digital TUI Simulation Environment}

A simulation environment for TUIs needs to fulfill several requirements in order to enable a complete evaluation of the system at an early stage and to fulfill the key properties of TUIs as described by Kim and Maher (Kim \& Maher 2008). Since tangible user interfaces are based on the linkage of the digital and physical domain, it is important that the simulation can simulate any kind of physical object, especially rigid body objects that are commonly used in many activities of daily living (ADL). A physics engine has to ensure that the digital objects 


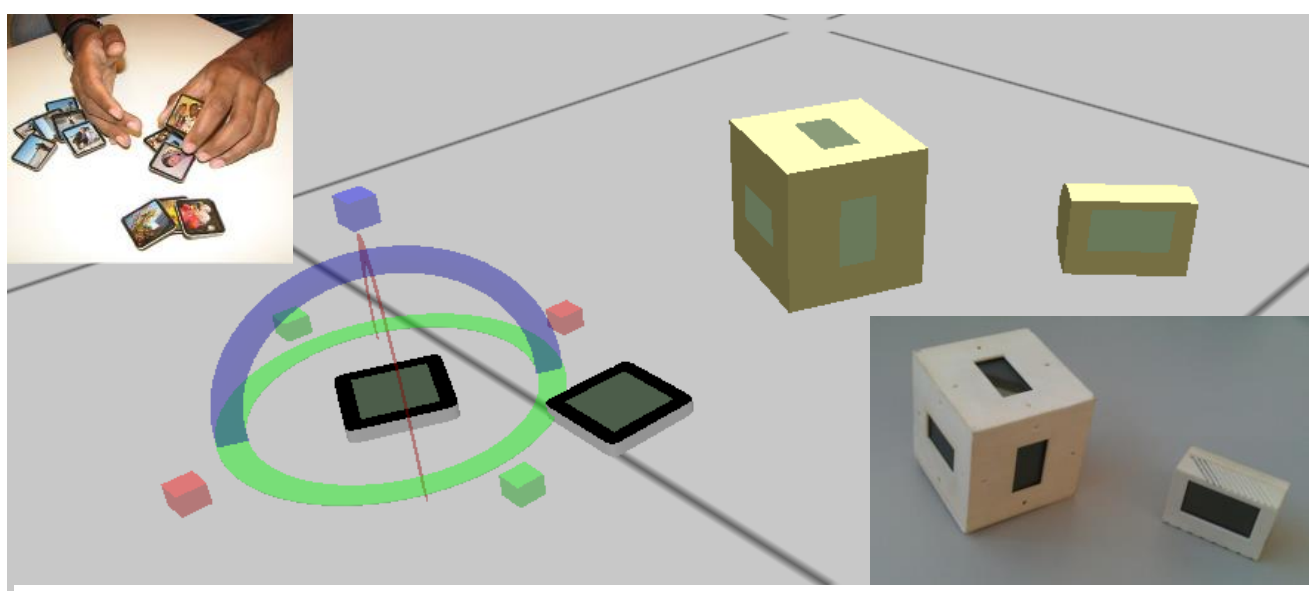

Figure 2: Digital TUIs (from left to right: two Sifteo cubes, an I/O cube and a ubiquitous presence system) and their real counterparts (images in the top left and bottom right corners). The left digital TUI is currently manipulated through the GUI. Gazebo allows for manipulation in all 6 degrees of freedom by applying force to the object.

behave like real physical ones. Besides the support for modeling objects of any arbitrary shape, it should further allow the use of realistic textures. Simple geometric shapes as well as complex TUIs should be supported. In order to allow an intuitive evaluation by the user, it has to offer a user-friendly $3 \mathrm{D}$ interface with the possibility to interact with the simulated objects and to explore the virtual environment. Another important factor is the connection of the simulation environment to a middleware that can actively support the TUI development. It would be useful to choose one that can be used for the virtual simulation as well as afterwards for a physical implementation.

\subsection{The ROS Middleware as TUI Middleware}

Based on the requirements analysis, we have chosen the Robot Operating System $(R O S)^{1}$ as middleware. ROS is one of the major middleware systems in the domain of robotics. Thus, an advantage of this middleware is that a huge set of drivers and applications is already available. The middleware already provides us with basic messages and communication protocols to transfer any kind of data between different nodes. For the digital development process, ROS does not depend on existing hardware. The integrated 3D visualization tool Gazebo allows evaluating the digital prototype in a high-fidelity virtual environment with realistic physical properties. The physical elements for the simulation can be modeled with all common 3D modeling tools, such as Blender ${ }^{2}$ or Cinema $4 D$. Gazebo's functionality can be easily extended through a well-documented API, which allows, e.g., adding new kinds of virtual sensors and actuators. User interaction is possible via a graphical user interface (GUI).

http://www.ros.org/, last accessed Mar. 27, 2014.

2 http://www.blender.org/, last accessed Mar. 12, 2014. 


\section{Examples of Digital Prototypes}

A collection of digital TUIs is depicted in Fig. 2. It shows (from left to right) two virtual Sifteo cubes (Merrill et al. 2012), a digital I/O cube and a digital TUI for a ubiquitous presence system (Kranz et al. 2006). The meshes have been created with the free 3D modeling software Blender. In order to allow physical simulation, the centers of gravity have been set to the objects' centers. Sizes and masses correspond to the respective physical objects. All of these TUIs are equipped with virtual accelerometers and virtual displays. The Sifteo cubes further have virtual proximity sensors and touch-sensitive screens (cf. Fig. 2 left). The communication system is based on the ROS middleware's communication infrastructure that can also be used by physical TUIs. The left Sifteo cube in Fig. 2 is selected for manipulation in Gazebo. The blocks and circles around the virtual object represent the six degrees of freedom (6DoF) that can be manipulated via the GUI. Besides mouse and keyboard input, the manipulation can also be performed with six-degrees-of-freedom (6DoF) technology such as 3Dconnexion's SpaceNavigator. This allows for a more realistic interaction with the 3D scene.

\section{References}

Diewald, S., Roalter, L., Möller, A., \& Kranz, M. (2011). Towards a Holistic Approach for Mobile Application Development in Intelligent Environments. Proc. MUM, ACM, pp. 73-80.

Holleis, P., Kranz, M., Gall, M., \& Schmidt, A. (2005). Adding Context Information to Digital Photos. Proc. ICDCSW, IEEE, pp. 536-542.

Ishii, H., \& Ullmer, B. (1997). Tangible Bits: Towards Seamless Interfaces Between People, Bits and Atoms. Proc. CHI, ACM, pp. 234-241.

Kim, M. J., \& Maher, M. L. (2008). The Impact of Tangible User Interfaces on Spatial Cognition During Collaborative Design. Design Studies, 29(3), 222-253.

Kranz, M., Holleis, P., \& Schmidt, A. (2006). Ubiquitous Presence Systems. Proc. SAC, ACM, pp. 19021909.

Merrill, D., Sun, E., \& Kalanithi, J. (2012). Sifteo Cubes. CHI EA, ACM, pp. 1015-1018.

Roalter, L., Möller, A., Diewald, S., \& Kranz, M. (2011). Developing Intelligent Environments: A Development Tool Chain for Creation, Testing and Simulation of Smart and Intelligent Environments. Proc. IE, ACM, pp. 214-221.

Sankaran, R., Ullmer, B., Ramanujam, J., Kallakuri, K., Jandhyala, S., Toole, C., \& Laan, C. (2009). Decoupling Interaction Hardware Design Using Libraries of Reusable Electronics. Proc. TEI ACM, pp. 331-337.

Serrano, M., Nigay, L., Lawson, J.-Y. L., Ramsay, A., Murray-Smith, R., \& Denef, S. (2008). The OpenInterface Framework: A Tool for Multimodal Interaction. CHI EA, ACM, pp. 3501-3506.

Van Laerhoven, K., Villar, N., Schmidt, A., Gellersen, H.-W., Hakansson, M., \& Holmquist, L. (2003). Pin\&Play: The Surface as Network Medium. IEEE Communications Magazine, 41(4), 90-95.

\section{Contact Information}

\{stefan.diewald, andreas.moeller, roalter\}@tum.de, matthias.kranz@uni-passau.de 